\title{
The effect of the apsidal motion on the light curve of the close binary HD $93205(03 \mathrm{~V}+\mathrm{O} 8 \mathrm{~V})^{\star}$
}

\author{
A. M. van Genderen ${ }^{\star}$ \\ Leiden Observatory, Postbus 9513, 2300 RA Leiden, The Netherlands \\ Received 25 June 2002/Accepted 28 August 2002

\begin{abstract}
The 1982-1985 photometry (VBLUW system) of the O3V+O8V close binary HD 93205 has been rediscussed because of new insights into its true nature and orbital changes. By comparing this data set with the one obtained by Antokhina motion became obvious: a phase shift between the two light curves and a small change of the shape. A phase-locked light due to absorption if the $\mathrm{O} 8$ star is seen through cooler inter-binary gas, e.g. the bow-shock between the two colliding winds.
\end{abstract} \\ et al. (2000) in 1993, and using the same ephemeris to construct the light curve in the phase diagram, the effect of the apsidal \\ variation in the $L$ passband (containing the higher Balmer lines) is clearly present in the 1982-1985 data set and is presumably
}

Key words. stars: variables: general - stars: binaries: close - stars: early-type - stars: individual: HD 93205

\section{Introduction}

HD $93205(\mathrm{O} 3 \mathrm{~V}+\mathrm{O} 8 \mathrm{~V})$ is the earliest known system for which an RV is known, of which the first was presented by Conti \& Walborn (1976). The binary is located in the very young open cluster Trumpler 16. The object was observed in 1982-1985 and discussed by van Genderen et al. (1985, Paper I; 1989, Paper IX), van Genderen (1985, Paper II) in the framework of a long lasting monitoring project to search for intrinsic light variations in massive stars ( $\alpha$ Cyg variables). Small light variations were found $(0.02)$ and a tentative quasi-period of $2^{\mathrm{d}} 17$ was determined. A photometric study in one narrow filter $(v)$ by Antokhina et al. (2000) in 1993 supported by RV observations from the literature, revealed that the light variations were due to an orbital revolution of two tidally distorted stars with a period of $6^{\mathrm{d}} 08075$

New radial velocity data were discussed by Morrell et al. (2001) leading to new orbital elements. They determined for the first time a period of apsidal motion caused by tidal and rotational distortions for the system amounting to $185 \mathrm{yr}$. A revision of the revolution period resulted in $6^{\mathrm{d}} 0803$.

Benvenuto et al. (2002) presented a model dependent method to calculate the individual masses with known apsidal rates. This is because the equation giving the rate of apsidal motion is a supplementary equation, so that the masses of the components can be computed if radii and internal structure constants are known from theoretical models.

The present paper discusses, apart from the brightness $V$, also the four colour indices of the 1982-1985 data sets, all in the $V B L U W$ system of Walraven (electronically available, see

\footnotetext{
* Table 1 is also available in electronic form at CDS via anonymous ftp to cdsarc.u-strasbg.fr $(130.79 .128 .5)$ or via http://cdsweb.u-strasbg.fr/cgi-bin/qcat?J/A+A/397/921 $\star \star$ e-mail: genderen@strw.leidenuniv.nl
}

Sect. 2). So far only the observations in the $V$ band relative to the comparison star were published (Papers I, IX). The phase diagrams of the 1982-1985 and 1993 data sets, the latter from Antokhina et al. (2000), are compared with each other using the spectroscopic zero point of the 1983 periastron passage as given by them, and the new period determined by Morrell et al. (2001). In this way, the effect by the apsidal motion can be investigated.

\section{The 1982-1985 observations}

The observations were made with the $90 \mathrm{~cm}$ Dutch telescope at the ESO, Chile, equipped with the $V B L U W$ simultaneous Walraven photometer. Further details on the observational technique can be found in Paper I. The data sets discussed in Paper I (1982-1983) and Paper IX (1985) were made with the same comparison star HD 93596 (B5 III, photometric data in Paper I), but with two different background positions. It turned out that the background light at the two postions slightly differed (due to very faint nebulosities). Small systematic differences between the relative brightness and colour indices of the two data sets result. In both data sets the program star HDE 330308 (O3.0 V(f); star turned out to be constant) was included in the observing sequence (Paper IX). In this second set another comparison star was used (HD 93712, A0 V, $V_{\mathrm{J}}=8.96$, $\left.(B-V)_{\mathrm{J}}=0.013\right)$ next to the two different sky backgrounds. In this way it was possible to check the constancy of the first comparison star and to derive the precise corrections for the differences in background light. These corrections ranged between 0.002 (in $V-B$ ) and 0.015 (in $B-U$ ). The 1985 data of HD 93205 were corrected for the difference in background light. 


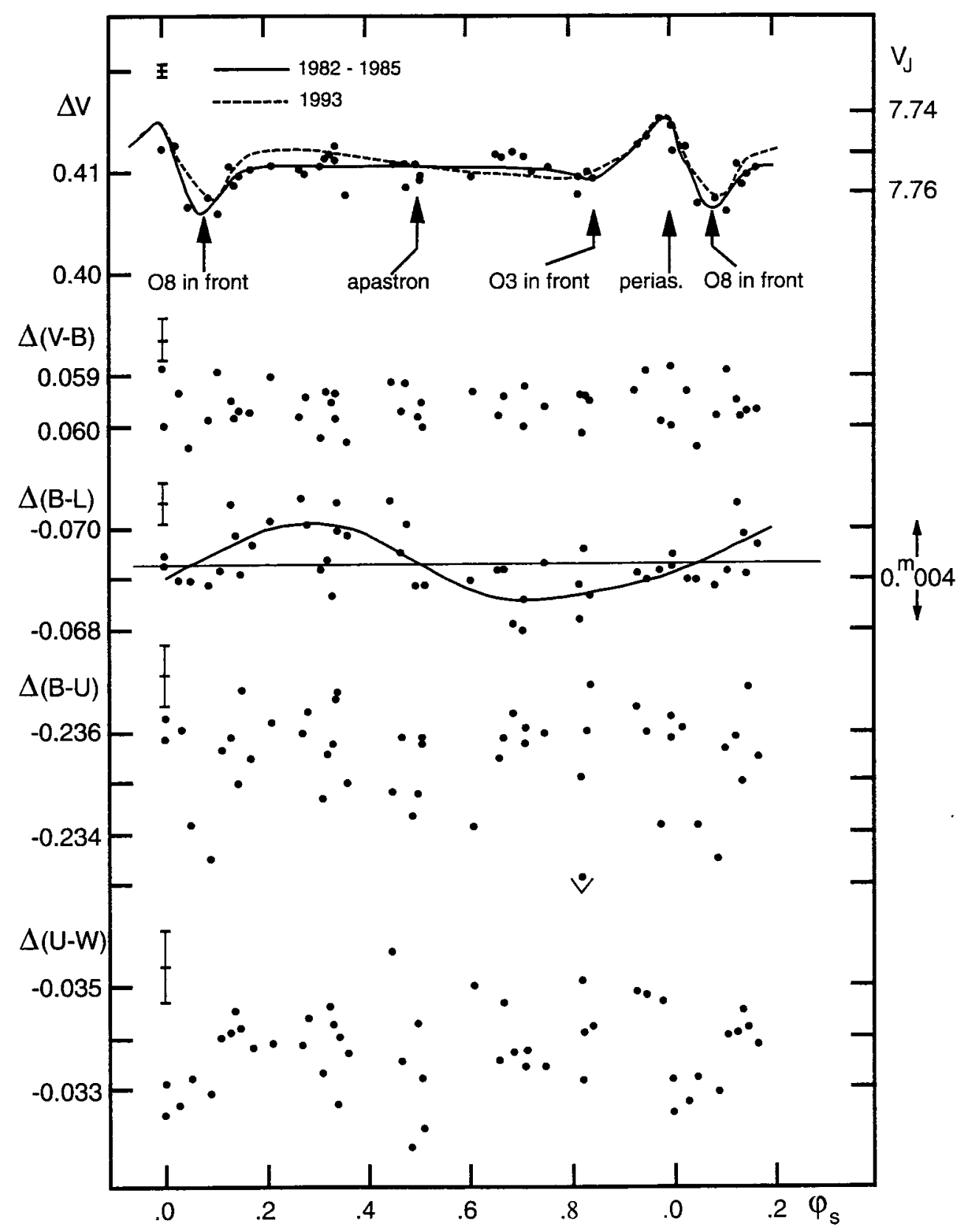

Fig. 1. The light and colour curves of HD 93205 in the $V B L U W$ system observed from 1982-1985 in log intensity scale (dots), as a function of the spectroscopic phase $\phi_{\mathrm{s}}$. The scale of the colours is five times larger than for the brightness $V$. Bright and blue are up. The error bars represents twice the mean error. The equivalent $V$ scale of the $U B V$ system in magnitides is indicated on the right. The dotted curve represents schematically the 1993 light curve by Antokhina et al. (2000), obtained in another visual filter, matched on the 1982-1985 light curve.

Each data point (nightly average) is an average of a sequence of generally eight observations of the variable alternating the comparison star(s) and sky background. Integration times per measurement were two times $30 \mathrm{~s}$. The duration of such a sequence was about half an hour.

Since in Papers I and IX only the relative brightnesses $V$ of HD 93205 were published (in the latter paper, already corrected), the electronically available Table 1 lists the brightness and colour indices in the natural $V B L U W$ photometric system. The equivalent values $V_{\mathrm{J}}$ of the $U B V$ system are added using a transformation formula. The new average brightness of HD 93205 is $V_{\mathrm{J}}=7.75$. Since the colour index $V-B$ does not show any significant variation, only the average computed $(B-V)_{\mathrm{J}}$ value is given here and is 0.082 . The transformation formulae are from Pel (1987) and are given in Paper IX.

\section{The light and colour curves}

For the construction of the phase diagram for brightness and colours, the following formula was used:

$\mathrm{HJD}=2445573.22+6.0803 E$.

The zeropoint represents a spectroscopically determined periastron passage in 1983 (Antokhina et al. 2000), thus, the same year as for most of our data points. These phases are 
Table 1. Log of brightness and colour indices of HD 93205 (1982-1985) in the VBLUW system (in log intensity scale) and the computed brightness $V_{\mathrm{J}}$ of the $U B V$ system.

\begin{tabular}{|c|c|c|c|c|c|c|}
\hline HJD - 2440000 & $V$ & $V-B$ & $B-U$ & $U-W$ & $B-L$ & $V_{\mathrm{J}}$ \\
\hline 5012.800 & -0.3486 & 0.0355 & -0.0427 & 0.0009 & -0.0120 & 7.754 \\
\hline 5013.718 & -0.3435 & 0.0360 & -0.0408 & 0.0003 & -0.0116 & 7.742 \\
\hline 5014.708 & -0.3498 & 0.0359 & -0.0417 & 0.0005 & -0.0123 & 7.758 \\
\hline 5015.806 & -0.3472 & 0.0354 & -0.0423 & 0.0004 & -0.0118 & 7.751 \\
\hline 5016.722 & -0.3498 & 0.0352 & -0.0411 & 0.0032 & -0.0125 & 7.758 \\
\hline 5020.726 & -0.3478 & 0.0356 & -0.0426 & 0.0009 & -0.0129 & 7.752 \\
\hline 5031.656 & -0.3460 & 0.0354 & -0.0432 & 0.0001 & -0.0115 & 7.748 \\
\hline 5032.607 & -0.3512 & 0.0359 & -0.0402 & 0.0021 & -0.0113 & 7.761 \\
\hline 5033.719 & -0.3481 & 0.0359 & -0.0427 & 0.0011 & -0.0130 & 7.753 \\
\hline 5034.771 & -0.3478 & 0.0352 & -0.0415 & -0.0007 & -0.0130 & 7.752 \\
\hline 5035.802 & -0.3489 & 0.0354 & -0.0408 & 0.0000 & -0.0114 & 7.755 \\
\hline 5036.646 & -0.3483 & 0.0357 & -0.0427 & 0.0016 & -0.0117 & 7.754 \\
\hline 5037.823 & -0.3451 & 0.0350 & -0.0427 & 0.0020 & -0.0114 & 7.746 \\
\hline 5038.819 & -0.3525 & 0.0350 & -0.0424 & 0.0010 & -0.0116 & 7.764 \\
\hline 5366.813 & -0.3516 & 0.0365 & -0.0409 & 0.0018 & -0.0114 & 7.762 \\
\hline 5367.764 & -0.3479 & 0.0351 & -0.0429 & 0.0011 & -0.0126 & 7.753 \\
\hline 5370.806 & -0.3488 & 0.0353 & -0.0425 & 0.0016 & -0.0110 & 7.755 \\
\hline 5372.750 & -0.3460 & 0.0354 & -0.0428 & 0.0023 & -0.0114 & 7.748 \\
\hline 5374.770 & -0.3505 & 0.0364 & -0.0417 & 0.0013 & -0.0123 & 7.759 \\
\hline 5376.757 & -0.3467 & 0.0351 & -0.0431 & 0.0013 & -0.0105 & 7.750 \\
\hline 5380.736 & -0.3458 & 0.0359 & -0.0435 & 0.0023 & -0.0127 & 7.748 \\
\hline 5381.743 & -0.3493 & 0.0361 & -0.0426 & 0.0028 & -0.0113 & 7.756 \\
\hline 5382.809 & -0.3470 & 0.0361 & -0.0428 & 0.0013 & -0.0104 & 7.750 \\
\hline 5383.774 & -0.3492 & 0.0356 & -0.0436 & 0.0008 & -0.0111 & 7.756 \\
\hline 5384.743 & -0.3438 & 0.0361 & -0.0430 & 0.0025 & -0.0119 & 7.742 \\
\hline 5385.753 & -0.3481 & 0.0358 & -0.0422 & 0.0012 & -0.0121 & 7.753 \\
\hline 5386.743 & -0.3466 & 0.0356 & -0.0425 & 0.0007 & -0.0111 & 7.750 \\
\hline 5387.750 & -0.3479 & 0.0359 & -0.0415 & 0.0007 & -0.0113 & 7.753 \\
\hline 5388.750 & -0.3466 & 0.0359 & -0.0422 & 0.0015 & -0.0116 & 7.750 \\
\hline 6140.770 & -0.3474 & 0.0359 & -0.0434 & 0.0010 & -0.0129 & 7.752 \\
\hline 6141.764 & -0.3487 & 0.0356 & -0.0425 & 0.0018 & -0.0117 & 7.755 \\
\hline 6142.778 & -0.3470 & 0.0355 & -0.0426 & 0.0030 & -0.0116 & 7.750 \\
\hline 6143.667 & -0.3506 & 0.0355 & -0.0418 & 0.0040 & -0.0113 & 7.760 \\
\hline 6144.760 & -0.3464 & 0.0349 & -0.0426 & 0.0040 & -0.0117 & 7.749 \\
\hline 6145.667 & -0.3487 & 0.0358 & -0.0436 & 0.0008 & -0.0115 & 7.755 \\
\hline 6149.757 & -0.3488 & 0.0362 & -0.0398 & -0.0001 & -0.0106 & 7.755 \\
\hline 6152.719 & -0.3478 & 0.0363 & -0.0414 & 0.0017 & -0.0116 & 7.752 \\
\hline 6158.614 & -0.3487 & 0.0355 & -0.0431 & 0.0006 & -0.0125 & 7.755 \\
\hline 6159.764 & -0.3478 & 0.0358 & -0.0426 & 0.0015 & -0.0119 & 7.752 \\
\hline
\end{tabular}

called in the present paper $\phi_{\mathrm{s}}$. The scale of the colours in Fig. 1 is five times larger than for the brightness (upper panel). At the right of the light curve the transformed $V$ of the $U B V$ system (with subscript $\mathbf{J}$ and in magnitude scale) is indicated. Error bars represent twice the average mean error per data point amounting to, in $\log$ intensity scale $(\times 0.0001): 5,4,4,6$ and 8 for $V, V-B, B-L, B-U$ and $U-W$, respectively (for magnitudes multiply with 2.5 ).

The various aspects of the binary are indicated below the light curve and were taken from the computer-simulated view of the binary in the plane of the sky by Antokhina et al. (2000). It happened, considering their binary model, that at $\phi_{\mathrm{s}}=0$, the passage through the periastron with maximum $V_{\text {rad }}$, both deformed stars are seen more or less from the side. The result is a light maximum. The computer-simulated binary model of Antokhina et al. (2000, their Fig. 5) is reproduced in Fig. 2 of the present paper, but with the $\mathrm{O} 3$ primary put at the origin. The six positions of the $\mathrm{O} 8$ secondary (full circles) are marked with their $\phi_{\mathrm{s}}$. Two other positions of the O8 secondary are indicated with dotted circles and will be discussed in Sect. 4.2.

Their model needs some revision in light of the improvements of the orbital elements determined by Morrell et al. (2001), but for our purpose it will do. Apart from the improvements, they found an apsidal motion with a period of 185 $\pm 16 \mathrm{yr}$. This means a shift of the periastron in the direction of the binary revolution of about $19^{\circ}$ over a time interval of about $10 \mathrm{yr}$, the time difference between the two photometric data sets.

The maximum of the continuous curve in Fig. 1 sketched through the 1982-1985 data points coincides with $\phi_{\mathrm{s}}=.0$ as it should, but the 1993 light maximum appears to lie ahead by .06. In Fig. 1 (upper panel) the 1993 curve (dotted) has 


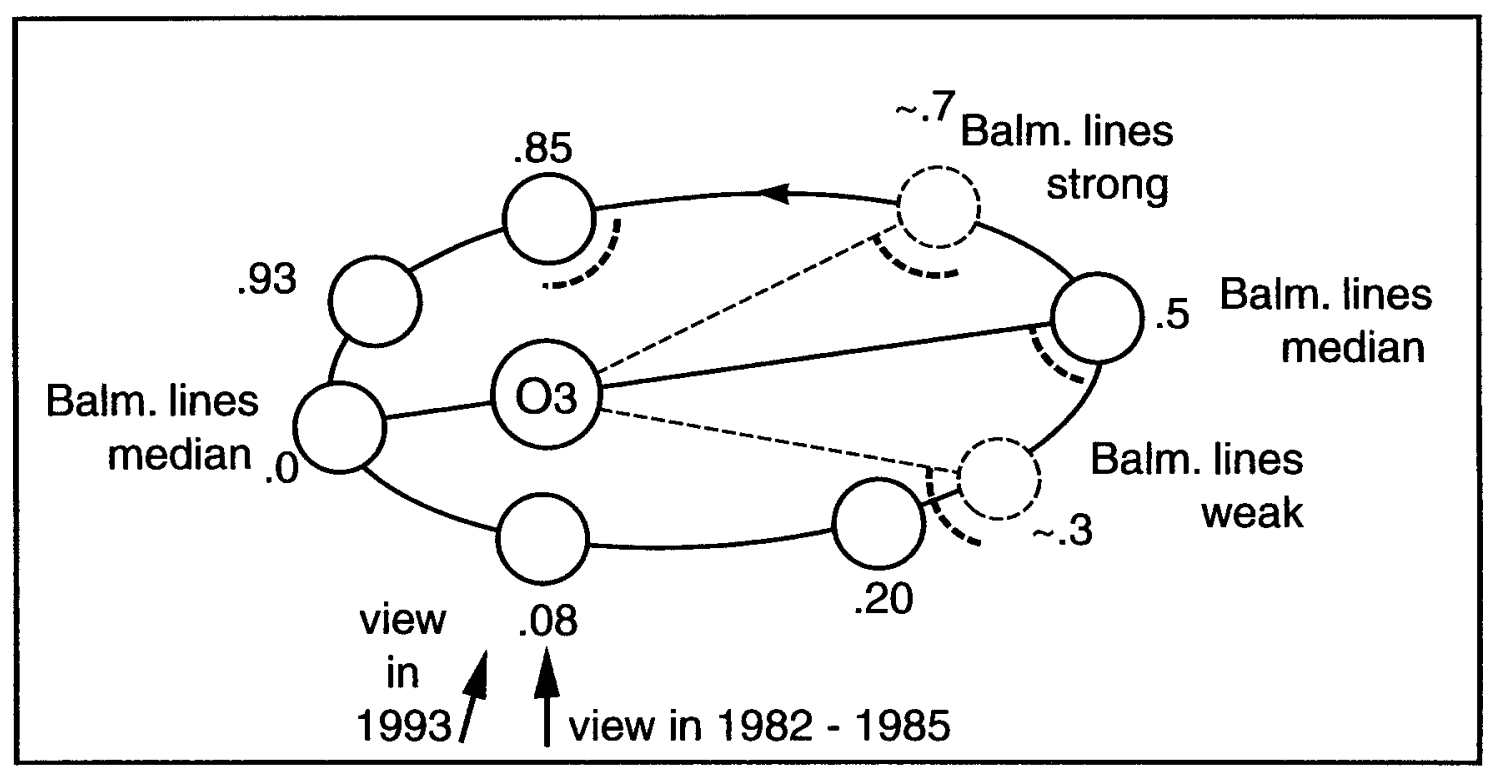

Fig. 2. The computer-simulated view of the binary for six phases $\left(\phi_{\mathrm{s}}\right)$, the $\mathrm{O} 8$ secondary represented by the full circles and the $\mathrm{O} 3$ primary put at the origin, in the plane of the sky, according to Antokhina et al.'s (2000) model. The two positions of the O8 secondary coinciding the extrema in the flux in the $L$ filter (containing the higher Balmer lines) are indicated by the two dotted circles.

been shifted back by .06 in order to match the one of $1982-$ 1985. This makes a comparison of the shapes easier (Sect. 4). Phases for the 1993 observations were also computed with a 1997 zeropoint for the maximum $V_{\text {rad }}$ of Morrell et al. (2001): 2450498.695 . It appears that with these phases the 1993 light curve nearly matches the 1982-1985 light curve. (Compared with the other zero point, the shift backwards amounts to .07). This result is consistent with the concept of a displaced periastron. Besides, .06 in phase corresponds to a rotation angle of $\sim 22^{\circ}$ which is close to the expected $19^{\circ}$ (Fig. 2).

It should be noted that a refinement of the period of Morrell et al. (2001) is not possible. Due to the displacement of the periastron, the observed projected maximum radial velocity might not coincide with the periastron passage anylonger. In fact, it is even not sure that this was truly the case for the data set 1982 1985 , but it has been adopted as such.

\section{Discussion}

\subsection{The light curves}

The extrema of the two light curves in Fig. 1 match reasonably. The total light amplitude amounts to 0.02 . The difference in brightness between the deep minimum $\left(\phi_{\mathrm{s}}=.08\right.$ and the shallow one $\left(\phi_{\mathrm{s}}=.85\right)$ amounting to $0 \mathrm{~m} 01$, is presumably due to the reflection effect of the $\mathrm{O} 8$ star when the $\mathrm{O} 3$ star lies in front (Fig. 2). Due to the shift of the periastron to the configuration "O8 in front" $\left(\phi_{\mathrm{s}}=.08\right)$, one would expect a steeper descending branch in 1993. In fact, a slight opposite effect can be seen, of which the reality is doubtful considering the scatter in both light curves. Obviously, the $19^{\circ}$ displacement of the periastron has not significantly altered the shape and the amplitudes of the extrema. According to the model of Morrell et al. (2001), the longitude of the periastron is somewhat larger than the one of Antokhina et al. (2000), thus, the periastron should point more in our direction than according to Fig. 2.
The only significant difference between the two light curves is the absence of a decreasing trend between $\phi_{\mathrm{s}} \sim 0.2$ and $\sim 0.8$ in the 1982-1985 light curve. There is more light at $\phi_{\mathrm{s}} \sim 0.2$ by about 0.004 in the 1993 light curve, while the brightness declines by $\sim 0$ m 007 until the shallow minimum. This decreasing trend led Antokhina et al. (2000) to experiment with models including a hot spot on one of the components. The decrease should then represent the disappearance of the spot from sight. While the resulting model light curves fit the decreasing trend satisfactorily, (their Figs. 7a,b), they were well aware that there is little physical ground for spots on these hot stars because of the absence of convective layers. Also, turbulent viscosity developing at periastron, causing tidal lag, was a reasonable alternative (their Fig. 7c). Any wind-related phenomenon, such as additional heating produced by $\mathrm{X}$-ray radiation of the bowshock formed by the two colliding winds on the surface of the closest star, was most unlikely.

A likely explanation could be as follows. In 1993 the viewing direction to the system makes a smaller angle (by $19^{\circ}$ ) with the periastron (Fig. 2). Consequently, the configuration "O8 in front" in 1993 lies closer to the periastron, resulting in a stronger deformation. Thus, more light can be expected around $\phi_{\mathrm{s}} \sim .2$ in 1993. Slightly before and in the configuration "O3 in front", the stars should show less deformation than in 1982-1985 because of the larger distance to the periastron. The 1993 light curve shows indeed less light around $\phi_{\mathrm{s}} \sim .7$. Whether the earlier start of the rising branch in 1993 at $\phi_{\mathrm{s}}=.8$ is significant cannot be said.

\subsection{The colour curves}

Figure 1 shows the four two-colour curves relative to the comparison star (scales in log intensity, with at the right of the $B-L$ curve an indication of the light amplitude in magnitude scale). In view of the size of the average mean error bars indicated 
at the left top of each diagram (m.e. $=\sigma / \sqrt{n}$ ), amounting to $\lessgtr 0$ m 002 , all, with the exception of the $B-L$, can be considered as scatter diagrams.

The $B-L$ shows a significant sine-like behaviour. A mean curve has been drawn through the data points having a total amplitude (from maximum to minimum) of 0.004 . The horizontal line represents the median colour. It appears that the blue part (above the line) is shorter (.45 in phase) than the red part (below the line; .55 in phase). Since the $V-B$ and $B-U$ are constant within the errors, the periodic variability of $B-L$ must be due to revolution modulation of the light in the $L$ band. This band contains the higher Balmer lines and the Balmer limit. Since the two stars are far too hot to show Balmer lines, we speculate that cooler inter-binary gas is responsible for the modulation by absorption with a total range of $0.4 \%$ in the flux received through the $L$ band. The brightness in $L$ has a maximum at $\phi_{\mathrm{s}} \sim .3$ (thus, Balmer lines weaker, therefore less absorption by cooler gas), and a minimum at $\phi_{\mathrm{s}} \sim .7$ (thus, Balmer lines stronger, therefore more absorption by cooler gas). The position of the O8 secondary at these moments are indicated by dotted circles in Fig. 2. The median values are reached around apastron and periastron, see Fig. 2.

A varying projection of an absorbing bowl-shaped interface between two colliding winds on the $\mathrm{O} 8$ star, as seen from the Earth, can offer an explanation for the phase-locked Balmer line modulation. E.g. at $\phi_{\mathrm{s}} \sim .7$, the absorption of the $\mathrm{O} 8 \mathrm{star}$ by the interface (thick dashed curve) has reached a maximum, thus, the Balmer lines are strong, flux in $L$ is lower, $B-L$ is reddest and the curve shows a minimum). It should be noted that the interface should lie closer to the 08 star than to the O3 star, since the wind of the latter is likely stronger and the interface might lag behind due to the revolution. During other configurations, such as the three other positions, the absorption is less and has obviously reached a minimum at $\phi_{\mathrm{s}} \sim .3$. This is peculiar: one would expect a minimum absorption around $\phi_{\mathrm{s}} \sim .08$ ("O8 star in front"). It is possible that due to the much shorter distance between the stars, the bow-shock is pressed all around and to the other side of the $\mathrm{O} 8$ star, forming a tail or wake, through which we see the star. This absorbing material should then be absent some time later, when the $\mathrm{O} 8$ star is at a further distance from the $\mathrm{O} 3$ star. It may also explain why the blue part of the $B-L$ curve (less absorption) lasts a shorter time than the red part.

\section{Conclusions}

The 1982-1985 photometric observations (VBLUW system) are rediscussed and tabulated in detail in view of the fact that the light variation appears not to be due to intrinsic variations, but due to the revolution of an $\mathrm{O} 3 \mathrm{~V}+\mathrm{O} 8 \mathrm{~V}$ binary. A comparison of these observations with those of Antokhina et al. (2000) made about $10 \mathrm{yr}$ later, showed not only a phase shift between the two data sets amounting to .06 (despite the use of an improved period determined by Morrell et al. 2001), but also small differences in the shape of the light curve. The explanation is that due to the apsidal motion found by Morrell et al. (2001), the orientation of the binary with respect to the observer has changed.

It appears that the light in the $L$ band, containing the higher Balmer lines and Balmer limit, shows a significant phase locked variation amounting to $0.4 \%$. This can be explained by the varying absorption effect due to cooler inter-binary gas, likely concentrated in a bow-shock between the two colliding winds.

Acknowledgements. I am much indebted to the referee for the constructive comments.

\section{References}

Antokhina, E. A., Moffat, A. F. J., Antokhin, I. I., et al. 2000, ApJ, 529,463

Benvenuto, O. G., Serenelli, A. M., Althaus, L. G., et al. 2002, MNRAS, 330, 435

Conti, P. S., \& Walborn, N. R. 1976, ApJ, 207, 502

van Genderen, A. M. 1985, A\&A, 151, 349 (Paper II)

van Genderen, A. M., Alphenaar, P., van der Bij, M. D. P., et al. 1985, A\&AS, 61, 213 (Paper I)

van Genderen, A. M., Bovenschen, H., Engelsman, E. C., et al. 1989, A\&AS, 79, 263 (Paper IX)

Morrell, N. I., Barbá, R. H., Niemela, V. S., et al. 2001, MNRAS, 326, 85

Pel, J. W. 1987, Internal Report Leiden Obs. 